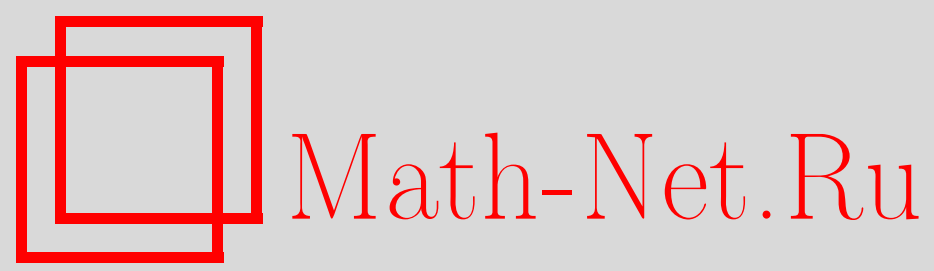

С. В. Архипов, О суммировании по Абелю обратного преобразования Фурье однородных функций в $R^{n}$, Вестник ТвГУ. Серия: Прикладная математика, 2019, выпуск 4, 98-107

DOI: https://doi.org/10.26456/vtpmk547

Использование Общероссийского математического портала Math-Net.Ru подразумевает, что вы прочитали и согласны с пользовательским соглашением

http: //www.mathnet.ru/rus/agreement

Параметры загрузки:

IP : 54.224 .60 .19

26 апреля 2023 г., 11:10:48 
УДК $517.521 .7,517.443$

\section{О СУММИРОВАНИИ ПО АБЕЛЮ ОБРАТНОГО ПРЕОБРАЗОВАНИЯ ФУРЬЕ ОДНОРОДНЫХ ФУНКЦИЙ В $R^{n}$}

Архипов С.B.

Тверской государственный университет, г. Тверь

Поступила в редакцию 03.11.2019, после переработки 20.12.2019.

Как известно, к наиболее часто употребляемым функциям на прямой относятся степенные функции. Многомерным аналогом степенных функций являются однородные, имеющие вид $\theta(\tau)|t|^{\alpha}$, в которых помимо параметра $\alpha$ присутствует произвольная функция на единичной сфере. При вычислении обратного преобразования Фурье этих функций имеются ограничения на порядок $\alpha$. Одним из приемов для улучшения сходимости является суммирование по Абелю. В статье получены формулы суммирования по Абелю для обратного преобразования Фурье однородных функций, имеющих вид $\theta(\tau)|t|^{\alpha}$, $\tau \in S^{n-1}=\left\{t \in \mathbb{R}^{n}:|t|=1\right\}$, для различных функциональных пространств на единичной сфере.

Ключевые слова: суммирование по Абелю, обратное преобразование Фурье, однородные функции.

Вестник ТвГУ. Серия: Прикладная математика. 2019. № 4. С. 98-107. https://doi.org/10.26456/vtpmk547

\section{Введение}

В настоящее время применяются различные подходы к вычислению преобразования Фурье однородных функций в $\mathbb{R}^{n}$

$$
F \theta(\tau)|t|^{\alpha}=\int_{\mathbb{R}^{n}} e^{i(x, t)} \theta(\tau)|t|^{\alpha} d t=\lim _{N \rightarrow \infty} \int_{|x| \leq N} e^{i(x, t)} \theta(\tau)|t|^{\alpha} d t,
$$

где $t=|t| \tau$. Отметим, что $F[\cdot](t)=(2 \pi)^{n} F^{-1}[\cdot](-t)$.

В статье [5], посвящённой вычислению характеристики по символу, показано, что $(1)$ в силу $L_{1}$ и $L_{2}$-теории является сходящимся для достаточно гладкой функции $\theta(\tau)$ при $-n<\alpha<\operatorname{fracn} 2$. Для вычисления этого интеграла при $\alpha \geq-\frac{n}{2}$ применяется явная регуляризация, использующая средние следов на единичной сфере. Для этого были введены p.f.-интегралы и отмечалось, что их сходимость обеспечивается достаточной гладкостью функций на сфере. В [9] предложен подход, основанный на преобразовании Фурье обобщённых функций в $\mathbb{R}^{n}$. В [1] используется контурное интегрирование по двойным петлям в комплексной плоскости. 
В книге [8] рассматривается суммирование интегралов по Абелю. Мы будем опираться на изложение метода суммирования Абеля из книги Стейна, Вейса [7]. Основываясь на этом подходе, вычислим обратное преобразование Фурье однородных функций.

Сформулируем основные результаты.

Теорема 1. Пусть $\theta(\tau) \in C^{\infty}\left(S^{n-1}\right)$ - пространство бесконечно дифберенцируемых функций на единичной сфере. Тогда при $\alpha>-n$

$$
\lim _{\varepsilon \rightarrow 0} \frac{1}{(2 \pi)^{n}} \int_{\mathbb{R}^{n}} e^{-i(x, t)} e^{-\varepsilon|t|} \theta(\tau)|t|^{\alpha} d t=\frac{2^{\alpha}}{\pi^{n / 2}} \sum_{l, j}(-i)^{l} \theta_{l j} \frac{\Gamma\left(\frac{\alpha+n+l}{2}\right)}{\Gamma\left(\frac{l-\alpha}{2}\right)} Y_{l j}(\tau) .
$$

Теорема 2. Пусть $\theta(\tau) \in L_{2}^{r}\left(S^{n-1}\right)-$ пространство дифферениируемых функций на сфере $S^{n-1}$, чви производные до порядка $r$ существуют и принадлежат пространству $L_{2}\left(S^{n-1}\right)$ суммируемых с квадратом функиий. Тогда (2) справедливо при $r \geq \alpha+\frac{n}{2}$ u $\alpha>-n$.

\section{1. Некоторые сведения из теории функций на сфере}

Рассмотрим однородные гармонические многочлены порядка $l: P_{l}(x), x \in \mathbb{R}^{n}$.

Определение 1. Будем называть сужение $P_{l}(x)$ на единичную сферу $S^{n-1}$

$$
|x|^{-l} P_{l}(x)=Y_{l}(\xi), \quad \xi=\frac{x}{|x|} \in S^{n-1}, \quad l=0,1,2, \ldots
$$

сферической гармоникой порядка l.

Лемма 1 ( [5], с.30). Пусть имеем произвольные сферические гармоники $Y_{l}(\xi)$ и $Y_{k}(\xi)$ разного порядка $k, l=0,1,2, \ldots, k \neq l$. Тогда справедливо следующее равенство

$$
\int_{S^{n-1}} Y_{l}(\xi) Y_{k}(\xi) d \xi=0
$$

Обозначим через

$$
L_{2}\left(S^{n-1}\right)=\left\{\theta(\xi):\left(\int_{S^{n-1}}|\theta(\xi)|^{2} d \xi\right)^{\frac{1}{2}}<\infty\right\}
$$

гильбертово пространство суммируемых с квадратом функций на сфере со скалярным произведением

$$
\int_{S^{n-1}} \theta_{1}(\xi) \theta_{2}(\xi) d \xi
$$

Зафиксируем в $L_{2}\left(S^{n-1}\right)$ ортонормированный базис из сферических гармоник порядка $l: Y_{l j}(\xi) ; l=0,1,2, \ldots ; j=1,2, \ldots, d(l)$, где $d(l)$ - размерность пространства сферических гармоник порядка $l$

$$
d(l)=\frac{(n+2 l-2) \Gamma(n+l-2)}{\Gamma(l+1) \Gamma(n-1)} .
$$


Лемма 2 ( $[5]$, с. 30$)$. Функиии $\left\{Y_{l j}(\xi)\right\}$ образуют полную ортонормированную систему функиий на сфере $S^{n-1}$.

Таким образом, каждую функцию из $L_{2}\left(S^{n-1}\right)$ можно представить с помощью сходящегося в среднем квадратичном ряда Фурье-Лапласа

$$
\theta(\xi)=\sum_{l=0}^{\infty} \sum_{j=1}^{d(l)} \theta_{l j} Y_{l j}(\xi)
$$

Коэффициенты разложения определяются по формулам

$$
\theta_{l j}=\int_{S^{n-1}} \theta(\xi) Y_{l j}(\xi) d \xi .
$$

Введём функциональные пространства дробной гладкости для функций из $L_{2}\left(S^{n-1}\right)$. Рассмотрим оператор Лапласа. Для его записи будем использовать сферические координаты:

$$
\begin{aligned}
\xi_{1} & =\cos \varphi_{1}, \\
\xi_{2} & =\sin \varphi_{1} \cos \varphi_{2}, \\
\xi_{3} & =\sin \varphi_{1} \sin \varphi_{2} \cos \varphi_{3}, \\
& \ldots \\
\xi_{n-2} & =\sin \varphi_{1} \ldots \sin \varphi_{n-3} \cos \varphi_{n-2}, \\
\xi_{n-1} & =\sin \varphi_{1} \ldots \sin \varphi_{n-3} \sin \varphi_{n-2} \cos \varphi_{n-1}, \\
\xi_{n} & =\sin \varphi_{1} \ldots \sin \varphi_{n-3} \sin \varphi_{n-2} \sin \varphi_{n-1},
\end{aligned}
$$

причём $0 \leq \varphi_{j} \leq \pi, j=1, \ldots, n-2,0 \leq \varphi_{n-1}<2 \pi$.

Лапласиан в $\mathbb{R}^{n}$, рассмотренный в сферических координатах, допускает следующее представление

$$
\begin{aligned}
\Delta & \equiv \sum_{j=1}^{n} \frac{\partial^{2}}{\partial x_{j}^{2}}=\Delta_{\rho}-\frac{1}{\rho^{2}} \Delta_{\xi}, \quad \text { где } \\
\Delta_{\rho} & =\frac{\partial^{2}}{\partial \rho^{2}}+\frac{n-1}{\rho} \frac{\partial}{\partial \rho}, \\
\Delta_{\xi} & =-\sum_{j=1}^{n-1} \frac{1}{q_{j}\left(\sin \varphi_{j}\right)^{n-j-1}} \frac{\partial}{\partial \varphi_{j}}\left(\sin ^{n-j-1}\left(\varphi_{j}\right) \frac{\partial}{\partial \varphi_{j}}\right), \\
q_{1} & =1, \\
q_{j} & =\prod_{k=1}^{j-1}\left(\sin \varphi_{k}\right)^{2}, \quad \text { при } j>1 .
\end{aligned}
$$

Оператор $\Delta_{\rho}$ называется радиальной частью $\Delta$, а $\Delta_{\xi}-$ сферическим оператором Лапласа или оператором Бельтрами-Лапласа. Будем обозначать через $\theta\left(\frac{x}{|x|}\right)$, $x \in \mathbb{R}^{n}$ продолжение функции $\theta(\xi)$ постоянным образом по радиальным направлениям $\xi \in S^{n-1}$. Понятно, что

$$
\Delta_{\xi} \theta(\xi)=\Delta \theta\left(\frac{x}{|x|}\right)
$$


в точках сферы и поэтому можно говорить о совпадении дифференциальных свойств на сфере и в пространстве.

Лемма 3 ( [3], с. 144). Собственными функииями оператора $\Delta_{\xi}$ являются сферические гармоники, причём

$$
\Delta_{\xi} Y_{l}(\xi)=l(l+n-2) Y_{l}(\xi), \quad l=0,1,2, \ldots
$$

Определение 2. Оператор, определённый равенством

$$
T \theta=\sum_{l, j} t_{l} \theta_{l j} Y_{l j}(\xi)
$$

будем называть мультипликаторным, а его спектр $\left\{t_{l}\right\}$ мультипликатором по сферическим гармоникам ([10], с. 510).

Рассмотрим мультипликаторный оператор $\left(E+\Delta_{\xi}\right)^{\frac{r}{2}}$ с мультипликатором $\left\{(1+l(l+n-2))^{\frac{r}{2}}\right\}$, где $E-$ единичный оператор.

Определение 3. Если $0<r<\infty$, то в пространство $L_{2}^{r}\left(S^{n-1}\right)$ будем включать те функиии $\theta(\xi)$, определённые на сфере, для которых

$$
\left(E+\Delta_{\xi}\right)^{\frac{r}{2}} \theta(\xi) \in L_{2}\left(S^{n-1}\right) .
$$

\section{2. Доказательство теорем}

Доказательство теоремы 1. Запишем формулу обратного преобразования Фурье однородной функции порядка $\alpha$ и сделаем замену переменной $r=|x||t|$ :

$$
\begin{aligned}
& F^{-1} \theta(\tau)|t|^{\alpha}=\frac{1}{(2 \pi)^{n}} \int_{\mathbb{R}^{n}} e^{-i(x, t)} \theta(\tau)|t|^{\alpha} d t= \\
&=\frac{1}{(2 \pi)^{n}|x|^{\alpha+n}} \int_{S^{n-1}} \int_{0}^{\infty} e^{-i(\xi, \tau) r} \theta(\tau) r^{\alpha+n-1} d r d \tau .
\end{aligned}
$$

Так как при $-n<\alpha<-\frac{n}{2}$ этот интеграл определяет обычную функцию (см. [5]), то можно просуммировать его не изменяя результата (см. [7], §1):

$$
F^{-1} \theta(\tau)|t|^{\alpha}=\lim _{\varepsilon \rightarrow 0} \frac{1}{(2 \pi)^{n}|x|^{\alpha+n}} \int_{S^{n-1}} \int_{0}^{\infty} e^{-i(\xi, \tau) r} e^{-\varepsilon r} \theta(\tau) r^{\alpha+n-1} d r d \tau .
$$

Применим во внутреннем интеграле формулу (3.381.4) из [2]:

$$
\int_{0}^{\infty} x^{\nu-1} e^{-\mu x} d x=\frac{1}{\mu^{\nu}} \Gamma(\nu), \quad \operatorname{Re} \mu>0, \quad \operatorname{Re} \nu>0 .
$$


Тогда

$$
\begin{aligned}
F^{-1} \theta(\tau)|t|^{\alpha}=\frac{\Gamma(\alpha+n)}{(2 \pi)^{n}|x|^{\alpha+n}} & \lim _{\varepsilon \rightarrow 0} \int_{S^{n-1}} \frac{\theta(\tau)}{(i(\xi, \tau)+\varepsilon)^{\alpha+n}} d \tau= \\
= & \frac{1}{(2 \pi)^{n}|x|^{\alpha+n}} \lim _{\varepsilon \rightarrow 0} \frac{\Gamma(\alpha+n)}{\varepsilon^{\alpha+n}} \int_{S^{n-1}} \frac{\theta(\tau)}{\left(\frac{i(\xi, \tau)}{\varepsilon}+1\right)^{\alpha+n}} d \tau .
\end{aligned}
$$

Вычислим отдельно выражение под знаком предела. Применим разложение в ряд справедливое при $|x|<1$ :

$$
(1+x)^{-\nu}=\sum_{k=0}^{\infty}(-1)^{k} \frac{\Gamma(\nu+k)}{\Gamma(\nu) \Gamma(k+1)} x^{k} .
$$

Будем иметь при $\varepsilon>1$

$$
\begin{aligned}
& I=\frac{\Gamma(\alpha+n)}{\varepsilon^{\alpha+n}} \int_{S^{n-1}} \frac{\theta(\tau)}{\left(\frac{i(\xi, \tau)}{\varepsilon}+1\right)^{\alpha+n}} d \tau= \\
&=\frac{1}{\varepsilon^{\alpha+n}} \int_{S^{n-1}} \sum_{k=0}^{\infty}(-1)^{k} \frac{\Gamma(\alpha+n+k)}{\Gamma(k+1)}\left(\frac{i(\xi, \tau)}{\varepsilon}\right)^{k} \theta(\tau) d \tau .
\end{aligned}
$$

Поменяем местами знаки суммирования и интегрирования. Обоснование проведём позже.

$$
I=\frac{1}{\varepsilon^{\alpha+n}} \sum_{k=0}^{\infty}(-i)^{k} \frac{\Gamma(\alpha+n+k)}{\varepsilon^{k} \Gamma(k+1)} \int_{S^{n-1}}(\xi, \tau)^{k} \theta(\tau) d \tau .
$$

Подставим разложение (3) для $\theta(\tau)$ в ряд Фурье-Лапласа по сферическим гармоникам под знак интеграла

$$
I=\frac{1}{\varepsilon^{\alpha+n}} \sum_{k=0}^{\infty}(-i)^{k} \frac{\Gamma(\alpha+n+k)}{\varepsilon^{k} \Gamma(k+1)} \sum_{l, j} \theta_{l j} \int_{S^{n-1}}(\xi, \tau)^{k} Y_{l j}(\tau) d \tau .
$$

Для вычисления интеграла воспользуемся формулой (26.80) из [4]:

$$
\int_{S^{n-1}}(\xi, \tau)^{k} Y_{l j}(\tau) d \tau=\frac{2^{1-k} \pi^{n / 2} \Gamma(k+1)}{\Gamma\left(\frac{l+n+k}{2}\right) \Gamma\left(1+\frac{k-l}{2}\right)} Y_{l j}(\xi),
$$

если $k-l=0,2,4, \ldots$, в остальньх случаях целочисленньх значений разности $k-l$ интеграл равен 0 .

Применяя последнюю формулу, получим

$$
I=\frac{1}{\varepsilon^{\alpha+n}} \sum_{k=0}^{\infty}(-i)^{k} \sum_{l, j} \theta_{l j} Y_{l j}(\xi) \frac{2^{1-k} \pi^{\frac{n}{2}} \Gamma(\alpha+n+k)}{\varepsilon^{k} \Gamma\left(\frac{l+n+k}{2}\right) \Gamma\left(1+\frac{k-l}{2}\right)} .
$$


Отметим, что если $\frac{k-l}{2}=-1,-2,-3, \ldots$, то $\frac{1}{\Gamma\left(1+\frac{k-l}{2}\right)}=0$. Введём новый индекс суммирования: $K=\frac{k-l}{2}$. Перепишем сумму ряда по $k$ и поменяем местами знаки суммирования:

$$
I=\frac{\pi^{\frac{n}{2}}}{\varepsilon^{\alpha+n}} \sum_{l, j} \theta_{l j} Y_{l j}(\xi) \sum_{K=0}^{\infty}(-i)^{2 K+l} \frac{2^{1-(2 K+l)} \Gamma\left(2\left(K+\frac{\alpha+n+l}{2}\right)\right)}{\varepsilon^{2 K+l} \Gamma\left(\frac{2 l+2 K+n}{2}\right) \Gamma(K+1)} .
$$

Преобразуем гамма-функцию в числителе по формуле двойного аргумента:

$$
I=\frac{\pi^{\frac{n-1}{2}} 2^{\alpha+n}}{\varepsilon^{\alpha+n}} \sum_{l, j}\left(\frac{-i}{\varepsilon}\right)^{l} \theta_{l j} Y_{l j}(\xi) \times \sum_{K=0}^{\infty}(-i)^{2 K} \frac{\Gamma\left(K+\frac{\alpha+n+l}{2}\right) \Gamma\left(K+\frac{\alpha+n+l+1}{2}\right)}{\varepsilon^{2 K} \Gamma\left(K+l+\frac{n}{2}\right) \Gamma(K+1)} .
$$

При $\varepsilon>1$ второй ряд является гипергеометрическим. Он равномерно и абсолютно сходится, когда модуль аргумента меньше единицы. Используя обозначение гипергеометрического ряда, получаем

$$
\begin{aligned}
I=\frac{\pi^{\frac{n-1}{2}} 2^{\alpha+n}}{\varepsilon^{\alpha+n}} \sum_{l, j}\left(\frac{-i}{\varepsilon}\right)^{l} \theta_{l j} Y_{l j}(\xi) & \frac{\Gamma\left(\frac{\alpha+n+l}{2}\right) \Gamma\left(\frac{\alpha+n+l+1}{2}\right)}{\Gamma\left(l+\frac{n}{2}\right)} \times \\
& \times{ }_{2} F_{1}\left(\frac{\alpha+n+l}{2}, \frac{\alpha+n+l+1}{2} ; l+\frac{n}{2} ;-\frac{1}{\varepsilon^{2}}\right) .
\end{aligned}
$$

Очевидно, что при $\varepsilon>1-\frac{1}{\varepsilon^{2}} \in(-1,0)$. Осуществим аналитическое продолжение гипергеометрического ряда в комплексную плоскость с разрезом по лучу $(1, \infty)$ с помощью преобразования $w=\frac{z}{z-1}$. (см. [2], 9.131.1):

$$
{ }_{2} F_{1}(a, b ; c ; z)=(1-w){ }_{2}^{a} F_{1}(a, c-b ; c ; w) .
$$

Тогда имеем

$$
\begin{aligned}
I=\frac{\pi^{\frac{n-1}{2}} 2^{\alpha+n}}{\varepsilon^{\alpha+n}} & \sum_{l, j}\left(\frac{-i}{\varepsilon}\right)^{l} \theta_{l j} Y_{l j}(\xi)\left(1-\frac{1 / \varepsilon^{2}}{1 / \varepsilon^{2}+1}\right)^{\frac{\alpha+n+l}{2}} \times \\
\times & \frac{\Gamma\left(\frac{\alpha+n+l}{2}\right) \Gamma\left(\frac{\alpha+n+l+1}{2}\right)}{\Gamma\left(l+\frac{n}{2}\right)} \times{ }_{2} F_{1}\left(\frac{\alpha+n+l}{2}, \frac{l-\alpha-1}{2} ; l+\frac{n}{2} ; \frac{1}{1+\varepsilon^{2}}\right) .
\end{aligned}
$$

Проводя преобразования, получаем справедливое при $\varepsilon>0$ выражение для $I$ :

$$
\begin{aligned}
I=\sum_{l, j}(-i)^{l} \theta_{l j} Y_{l j}(\xi)\left(\frac{1}{1+\varepsilon^{2}}\right)^{\frac{\alpha+n+l}{2}} & \times \frac{\Gamma\left(\frac{\alpha+n+l}{2}\right) \Gamma\left(\frac{\alpha+n+l+1}{2}\right)}{\Gamma\left(l+\frac{n}{2}\right)} \times \\
& \times{ }_{2} F_{1}\left(\frac{\alpha+n+l}{2}, \frac{l-\alpha-1}{2} ; l+\frac{n}{2} ; \frac{1}{1+\varepsilon^{2}}\right) .
\end{aligned}
$$

Подставляя полученное выражение в (4) и переходя к пределу, мы придём к

$$
\begin{aligned}
F^{-1} \theta(\tau)|t|^{\alpha}==\frac{2^{\alpha+n}}{\pi^{\frac{n+1}{2}}|x|^{\alpha+n}} \sum_{l, j}(-i)^{l} \theta_{l j} Y_{l j}(\xi) \frac{\Gamma\left(\frac{\alpha+n+l}{2}\right) \Gamma\left(\frac{\alpha+n+l+1}{2}\right)}{\Gamma\left(l+\frac{n}{2}\right)} \times & \\
& \times{ }_{2} F_{1}\left(\frac{\alpha+n+l}{2}, \frac{l-\alpha-1}{2} ; l+\frac{n}{2} ; 1\right) .
\end{aligned}
$$


Применим формулу для значения гипергеометрической функции в единице (см. [2], 9.122.1). После преобразований, получаем

$$
F^{-1} \theta(\tau)|t|^{\alpha}=\frac{2^{\alpha}}{\pi^{\frac{n}{2}}|x|^{\alpha+n}} \sum_{l, j}(-i)^{l} \frac{\Gamma\left(\frac{l+\alpha+n}{2}\right)}{\Gamma\left(\frac{l-\alpha}{2}\right)} \theta_{l j} Y_{l j}(\xi) .
$$

В последнем выражении получен мультипликаторный оператор, причём его спектр имеет порядок

$$
m_{l}=\frac{\Gamma\left(\frac{l+\alpha+n}{2}\right)}{\Gamma\left(\frac{l-\alpha}{2}\right)} \sim l^{\alpha+\frac{n}{2}} \quad \text { при } l \rightarrow \infty .
$$

Так как $\theta(\tau) \in C^{\infty}\left(S^{n-1}\right)$, то по теореме 4.8 из [5]

$$
\theta_{l j}=O\left(l^{-k}\right) \quad \text { для всех } k>0 .
$$

В силу этого получим абсолютно и равномерно сходящийся ряд в (5). Это обосновывает перемены знаков суммирования и интегрирования.

Исходное предположение $-n<\alpha<-\frac{n}{2}$ можно расширить в область $\alpha>0$, $\alpha \neq 1,2,3, \ldots$ в силу аналитичности по $\alpha$ правой части в $(2)$.

Доказательство теоремы 2. Все произведённые вычисления в теореме 1 остаются в силе. Исследуем на сходимость ряд Фурье-Лапласа в (5).

Необходимо обеспечить достаточный порядок гладкости полученной функции на сфере. Мультипликатор $m_{l}$ соответствует операции дифференцирования на сфере порядка $\alpha+\frac{n}{2}$. Для того, чтобы ряд был сходящимся в $L_{2}\left(S^{n-1}\right)$ необходимо потребовать, чтобы $\theta(\tau) \in L_{2}^{r}\left(S^{n-1}\right)$, где $r \geq \alpha+\frac{n}{2}$.

Отметим, что имеет место теорема вложения (см. [1], лемма 1.7):

$$
L_{2}^{r}\left(S^{n-1}\right) \subset C^{k}\left(S^{n-1}\right) \text { при } r \geq k+\frac{n}{2} .
$$

Таким образом, ряды в (5) абсолютно и равномерно сходятся и их сумма принадлежит пространству $C^{k}\left(S^{n-1}\right)$, если $r \geq k+\alpha+n$.

\section{Заключение}

Для получения разложений в ряд плотностей многомерных устойчивых распределений необходимо находить обратное преобразование Фурье однородных функций в $\mathbb{R}^{n}$. При вычислении обратного преобразования Фурье этих функций имеются ограничения на порядок $\alpha$. Одним из приемов для улучшения сходимости является суммирование по Абелю.

В статье получены формулы суммирования по Абелю для обратного преобразования Фурье этих функций, имеющих вид $\theta(\tau)|t|^{\alpha}, \tau \in S^{n-1}=\left\{t \in \mathbb{R}^{n}:|t|=1\right\}$, для различных функциональных пространств на единичной сфере. 


\section{Список литературы}

[1] Архипов С.В. Разложения плотности строго устойчивых распределений в $\mathbb{R}^{n}$ в случае абсолютно непрерывной спектральной меры: Дис. ... канд. физ.-мат. наук. 1989. $102 \mathrm{c.}$

[2] Градштейн И.С., Рыжик И.М. Таблицы интегралов, сумм, рядов и произведений. М.: Физматлит, 1963. 1100 с.

[3] Михлин С.Г. Многомерные сингулярные интегралы и интегральные уравнения. М.: Физматлит, 1962. 254 с.

[4] Самко С.Г. Гиперсингулярные интегралы и их приложения. Ростов: Изд-во Ростов. ун-та, 1984. 208 с.

[5] Самко С.Г. Обобщённые риссовы потенциалы и гиперсингулярные интегралы с однородными характеристиками, их символы и обращение // Труды Математического института им. В.А. Стеклова Академии наук СССР. 1980. Т. 156. C. $152-222$.

[6] Самко С.Г., Килбас А.А., Маричев О.И. Интегралы и производные дробного порядка и некоторые их приложения. Минск: Наука и техника, 1987. 688 с.

[7] Стейн И., Вейс Г. Введение в гармонический анализ на евклидовых пространствах. М.: Мир, 1974. 333 с.

[8] Bochner S. Harmonic analysis and the theory of probability. Berkeley: University of California Press, 1955. 176 p.

[9] Lemoine C. Fourier transforms of homogeneous distributions // Annali della Scuola normale superiore di Pisa. Classe di scienze. 1972. Vol. 26, № 1. Pp. 11-149.

[10] Rubin B. Introduction to Radon Transform: with elements of fractional calculations and harmonic analysis. 2015. 576 p.

\section{Образец цитирования}

Архипов С.В. О суммировании по Абелю обратного преобразования Фурье однородных функций в $R^{n} / /$ Вестник ТвГУ. Серия: Прикладная математика. 2019. № 4. C. 98-107. https://doi.org/10.26456/vtpmk547

\section{Сведения об авторах}

\section{1. Архипов Сергей Викторович}

доцент кафедры математической статистики и системного анализа Тверского государственного университета.

Россия, 170100, г. Тверь, ул. ЖКлябова, д. З3, ТвГУ. 


\title{
THE ABEL SUMMATION OF THE INVERSE FOURIER TRANSFORM OF THE HOMOGENEOUS FUNCTIONS IN $R^{n}$
}

\author{
Arkhipov Sergey Viktorovich \\ Associate Professor at the Department of Mathematical Statistics and System \\ Analysis, Tver State University \\ 170100, Russia, Tver, 33 Zhelyabova str., TverSU.
}

\begin{abstract}
Received 03.11.2019, revised 20.12.2019.
As is well known, the most commonly used functions on a line are powerful functions. A multidimensional analogue of power functions is homogeneous functions, which look like $\theta(\tau)|t|^{\alpha}$ and have an arbitrary function on a unit sphere additionally to the parameter $\alpha$. The inverse Fourier transform for these functions results in restrictions for an order of $\alpha$. One approach to improve convergence is Abel summation. Abel summation formulas for inverse Fourier transform of homogeneous functions have been derived in the article, which look like $\theta(\tau)|t|^{\alpha}, \tau \in S^{n-1}=\left\{t \in \mathbb{R}^{n}:|t|=1\right\}$ for various function spaces on a unit sphere.
\end{abstract}

Keywords: Abel summation formula, inverse Fourier transform, homogeneous functions.

\section{Citation}

Arkhipov S.V., "The Abel summation of the inverse Fourier transform of the homogeneous functions in $R^{n}$ ", Vestnik TvGU. Seriya: Prikladnaya Matematika /Herald of Tver State University. Series: Applied Mathematics], 2019, № 4, 98-107 (in Russian). https://doi.org/10.26456/vtpmk547

\section{References}

[1] Arkhipov S.V., Razlozheniya plotnosti strogo ustojchivykh raspredelenij $v \mathbb{R} n v$ sluchae absolyutno nepreryvnoj spektralnoj mery, PhD Thesis, 1989 (in Russian), 102 pp.

[2] Gradshtejn I.S., Ryzhik I.M., Tablitsy integralov, summ, ryadov i proizvedenij [Table of Integrals, Series, and Products], Fizmatlit Publ., Moscow, 1963 (in Russian), $1100 \mathrm{pp}$.

[3] Mikhlin S.G., Mnogomernye singulyarnye integraly i integralnye uravneniya [Multidimensional Singular Integrals and Integral Equations], Fizmatlit Publ., Moscow, 1962 (in Russian), 254 pp.

[4] Samko S.G., Gipersingulyarnye integraly i ikh prilozheniya [Hypersingular Integrals and Their Applications], Rostov University Press, Rostov, 1984 (in Russian), 208 pp. 
[5] Samko S.G., "Obobshchyonnye rissovy potentsialy i gipersingulyarnye integraly s odnorodnymi kharakteristikami, ikh simvoly i obrashchenie", Trudy Matematicheskogo instituta im. V.A. Steklova Akademii nauk SSSR [ Proceedings of the Steklov Institute of Mathematics], 156 (1980), 152-222 (in Russian).

[6] Samko S.G., Kilbas A.A., Marichev O.I., Integraly i proizvodnye drobnogo poryadka $i$ nekotorye ikh prilozheniya [Fractional integrals and derivatives: theory and applications], Nauka i Tekhnika, Minsk, 1987 (in Russian), 688 pp.

[7] Stejn I., Vejs G., Vvedenie v garmonicheskij analiz na evklidovykh prostranstvakh [Introduction to Fourier Analysis on Euclidean Spaces], Mir Publ., Moscow, 1974 (in Russian), 333 pp.

[8] Bochner S., Harmonic analysis and the theory of probability, University of California Press, Berkeley, 1955, 176 pp.

[9] Lemoine C., "Fourier transforms of homogeneous distributions", Annali della Scuola normale superiore di Pisa. Classe di scienze, 26:1 (1972), 11-149.

[10] Rubin B., Introduction to Radon Transform: with elements of fractional calculations and harmonic analysis, 2015, $576 \mathrm{pp}$. 